\title{
Comunicación icónica y gestual en Análisis Matemático
}

\author{
Miguel Delgado Pineda \\ Dep. Matemáticas Fundamentales. Facultad de Ciencias. \\ Universidad Nacional de Educación a Distancia UNED \\ Presentado en eXIDO16 (2016)

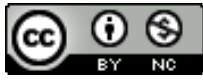

\begin{abstract}
Resumen
En este trabajo se presenta un proyecto de innovación docente que facilita a los estudiantes la adquisición o consolidación del concepto de función real de variable real. Los conceptos básicos tratados en este proyecto, función, variable y dominio..., suelen ser transmitidos priorizando el registro de la representación simbólica y el registro de la representación gráfica, sin enfatizar en el resto de registros de representación enumerados por Duval y otros investigadores. El diseño del proyecto se basa en presentar un juego donde las expresiones corporales mudas de los jugadores, estudiantes y profesor, son esenciales. Este juego puede ser resumido con dos preguntas: "¿qué función hago? y ¿qué función ves?”, o con el eslogan “mira e interpreta mis gestos matemáticos”, puesto que las funciones son presentadas mediante una comunicación gestual, es decir, toda una secuencia de gestos de un emisor a un receptor basado en un acercamiento visual antes de un acercamiento sintáctico y semántico de alguno de los posibles registros de representación simbólico de una función. La forma que los gestos reiterados suelen ser cristalizados en un conjunto de gestos finales de secuencia. Además, estos gestos finales permiten generar una serie de iconos que constituyen un nuevo registro de representación de clase funcional.

Las reglas del juego tan sólo requieren que el estudiante y el profesor utilicen, inicialmente, su cuerpo en sustitución de la pizarra o del ordenador, para ejercitar los gestos. En el aprendizaje, cada estudiante es una de las piezas del juego, y pueden actuar en forma individual o en forma colectiva. Los gestos sustituyen a las palabras, la secuencia gestual constituyen una sintaxis, y los iconos generados generan una semántica relativa al concepto de función. Lo vago que pudiera ser un gesto se perfila, finalmente, con un programa de representación gráfica de funciones en la pantalla de un ordenador en segunda instancia. El grafico del ordenador facilita adecuar y ajustar los iconos paradigma correspondiente a cada una de las funciones tratadas. Este juego permite generar en el estudiante un pensamiento funcional más allá del pensamiento numérico avanzado, puesto que una función es memorizada como un icono o como un conjunto de gestos.
\end{abstract}

Palabras clave: Concepto de función, Innovación Matemática Educativa, Comunicación no verbal, Formación del Profesorado.

\section{Introducción}

Nuestro trabajo se centra en no intentar generalizar procesos de la Enseñanza Secundaria a la Enseñanza Universitaria, si no en generar un cierto lenguaje que tanto el estudiante de secundaria como el estudiante universitario sepan emplear, por ello, nos centramos en el concepto de función de una variable real. Quizás el concepto básico del Análisis Matemático sea el concepto de función, y éste es uno de los objetos 
matemáticos más tratados en la literatura del Análisis Matemático o Cálculo, tanto a nivel educativo como didáctico.

En el supuesto de que le atribuyéramos capacidad de diálogo a dos funciones cualesquiera, quizás podríamos escuchar que ellas hicieran referencia a la frase de $\mathrm{N}$. Maquiavelo (El Príncipe):

Pocos ven lo que somos, pero todos ven lo que aparentamos.

Quizás sea el concepto de función un paradigma de la naturaleza de los objetos del Análisis Matemático, naturaleza que queda bien retratada en la siguiente frase (Frases y Citas, http://akifrases.com) atribuida a James Clerk Maxwell:

Todas las ciencias matemáticas se basan en las relaciones entre las leyes exactas, es reducir los problemas de la naturaleza a la determinación de las cantidades mediante operaciones con números.

Destacamos que en este párrafo, los términos: ciencia, naturaleza, física y matemática, constituyen el marco de referencia del trabajo intelectual humano. Los términos relación, ley, números, operación y cantidad representan los objetos básicos definidos en el marco matemático. Sin embargo, los términos problema, determinación y reducción establecen los constructos matemáticos con los que se manipulan esos objetos básicos.

Este concepto, el de función, aparece en los primeros temas a tratar en los libros de texto de Análisis Matemático o Cálculo, y, en muchos programas educativos, y , también, aparece en el nivel secundaria (NCTM, 2012). Además, dicho concepto es uno de los más analizados en la investigación educativa matemática (Kleiner, 1989; Luzín, 1998; Mesa y Villa, 2007; Mesa, 2004...).

La concepción de función reflejada en los libros de texto genera una dificultad didáctica al estudiante (Mesa y Villa 2007; Mesa 2004) por que presenta un carácter generalista. Esta dificultad puede ser evitada, al menos, para una gran parte de funciones, que es lo que llevamos a cabo desde nuestro proyecto innovador. Entendemos que variar los patrones habituales establecidos en los libros de texto tiene su dificultad profesional. Esto mismo ocurre con los patrones empleados por muchos profesores en sus prácticas docentes aun cuando tratan las funciones más elementales. Sin embargo, creemos que debemos pensar de forma creativa para permitirnos diseñar otros modelos educativos del concepto, que catalogamos como un modelo innovador.

Si bien, no es modificable el concepto actual de función, al menos se puede elegir un proceso educativo distinto para favorecer el aprendizaje del estudiante. En nuestro caso, el cambio para el aprendizaje se genera haciendo uso de técnicas comunicativas corporales, de modo que el estudiante adquiera de forma participativa (no pasiva) los elementos básicos (iconos), en cuanto a la forma y al modelo. En el proyecto establecemos una sintaxis y una semántica de gestos y de posicionamientos icónicos, y el foco del proceso se sitúa en el aprendizaje del estudiante, dando una importancia relativa al método de enseñanza empleado. Esto nos permite consensuar entre profesores y estudiantes varios puntos importantes, desde el juego de formas que se emplean hasta el mecanismo de comunicación multilateral, de todos a todos. No nos permitimos que la inercia de la costumbre y las asentadas culturas docentes matemáticas nos atenacen para repetir esquemas; supuestamente gastados y sospechosamente inoperantes. 
Tampoco nos podemos permitir que el estudiante huya del concepto de función sin que reconozca la dificultad de su aprendizaje y la extensión que tiene. El estudiante debe conocer que la dificultad de tratar de definir este concepto con precisión hasta llegar a una definición tal y como la actual, originó grandes controversias en la historia de la matemática y entre matemáticos de la misma época. En ciertos momentos históricos, esta dificultad fue un impedimento serio en el desarrollo del Cálculo Matemático de varias épocas. Este desarrollo nos hace entender que la concepción de función usual, de los profesores actuales, es consecuencia del desarrollo histórico del concepto, a los problemas que emergen en la historia y del posicionamiento filosófico sobre lo que se consideran los fundamentos de la Matemática (Mesa, 2004). Principalmente, esos desarrollos de fundamentación que impulsaron los matemáticos desde 1900.

Facilitar un cambio eficaz requiere voltear patrones clásicos y pensar de una forma creativamente de modo lateral (parcial) para tener posibilidades de generar otros esquemas mentales. Quizás esta conjunción de acciones es lo que llamamos innovación. Sin duda, para orientar al estudiante el profesor puede tener la necesidad de navegar por una lista de metodologías e ingenierías didácticas. También puede necesidad de intuir todas las protocolos de navegación y utilizar las múltiples herramientas didácticas disponibles para que el estudiante llegue al estado de saber. Tener necesidades de conocimientos didácticos específicos no nos puede hacer sin olvidar que algunas veces no es necesario explicar todo al estudiante, pues él debe tener una actuación activa y hay que darle la posibilidades de tener que tomar decisiones e interpretar los signos que aprecia. En "mensaje perfecto" no suele tener la componente empática que requiere el aprendizaje de estudiante. Ante un mensaje de ese tipo, el estudiante puede creer que nunca podrá generar un mensaje similar, así pues, memorizar el mensaje suele ser su respuesta de esfuerzo mínimo.

Ha de tenerse en cuenta los conocimientos que ya posee el estudiante e intentar actualizar o extender aquellos conceptos que pudieran generar una dificultas didáctica ante nuevos aprendizajes. Con el siguiente texto queremos mostrar un paradigma textual de los que queremos decir. Un texto en el que no se muestra la codificación utilizada en la confección del mensaje, sin embargo, el estudiante puede interpretarlo con cierta facilidad.

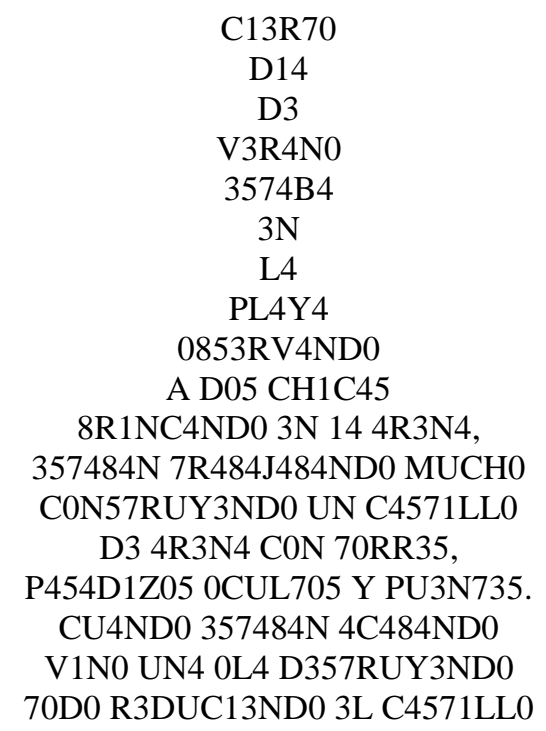


4 UN M0N70N D3 4R3N4 Y 35PUM4.

P3N53 9U3 D35PU35 DE 74N70 35FU3RZ0 L45 CH1C45 C0M3NZ4R14N 4 L10R4R, P3R0 3N V3Z D3 350, C0RR13R0N P0R L4 P14Y4 R13ND0 Y JU64ND0 Y C0M3NZ4R0N 4 C0N57RU1R 07R0 C4571LL0; C0MPR3ND1 9U3 H4814 4PR3ND1D0 UN4 6R4N L3CC10N; 64574M05 MUCH0 713MP0 D3 NU357R4 V1D4 C0N57RUY3ND0 4L6UN4 C054 P3R0 CU4ND0 M45 74RD3 UN4 0L4 LL364 4 D357RU1R 70D0, S010 P3RM4N3C3 L4 4M1574D, 3L 4M0R Y 3L C4R1Ñ0, Y L45 M4N05 D3 49U3LL05 9U3 50N C4P4C35 D3 H4C3RN05 50NRR31R.

Basta decir que si se mira el texto minuciosamente fijándose en cada uno de los símbolos, no lo entenderemos. Pues parece estar escrito en un idioma desconocido. Sin embargo, si creemos que es un texto en lengua española e intentamos leerlo, después de un poco tiempo, lo leeremos y entenderemos. Esto es debido a que nuestro cerebro aceptará rápidamente ciertas analogías y cambiará cada número por el carácter alfabético correspondiente.

Otro paradigma que afecta al sentido de la vista puede hacernos entender lo que estamos diciendo. Para ello basta mirar la figura 1. Es innecesario la descripción del cuadro La habitación de Arles de V. van Gogh y sin embargo se entiende lo que describe.

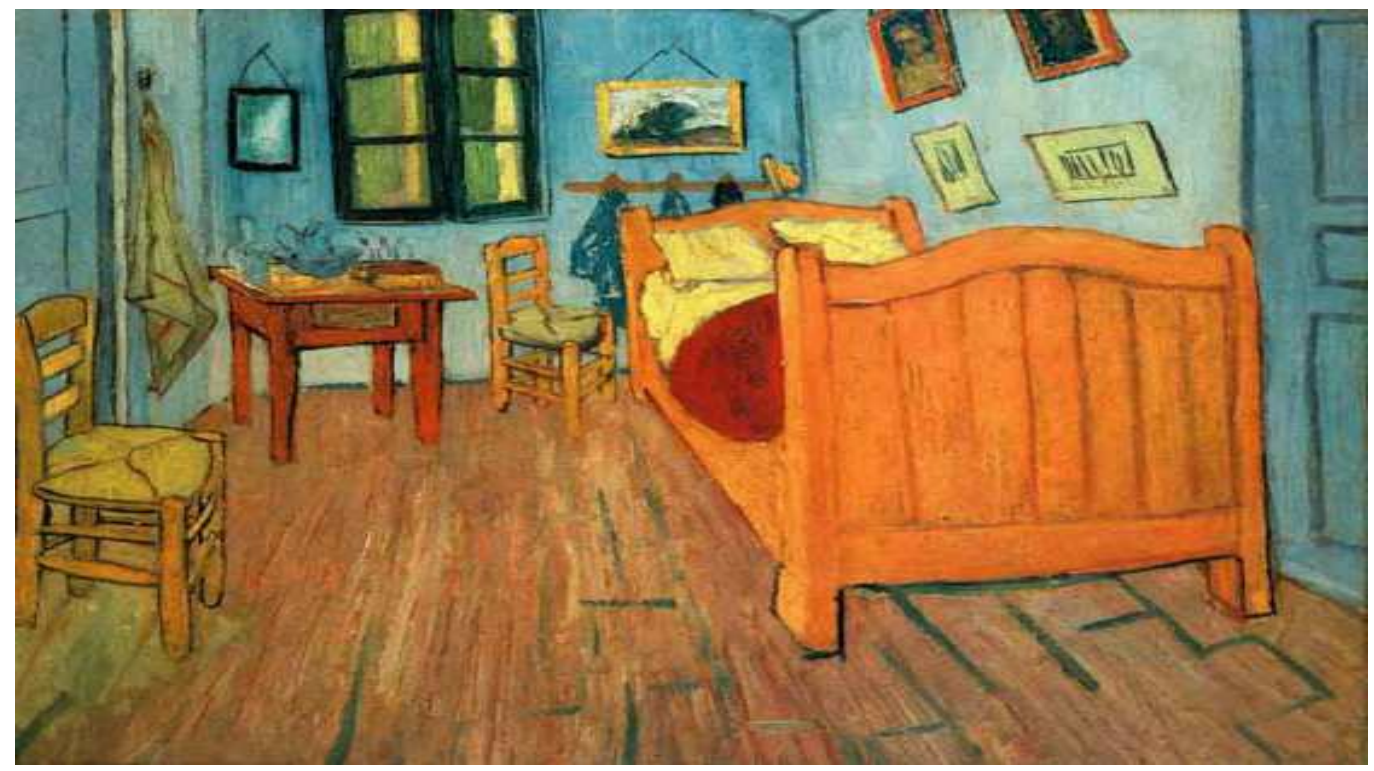

Figura 1: La habitación de Van Gogh en Arles (Musée d'Orsay).

Si nos fijamos bien podemos advertir que es una imagen sin ningún segmento rectilíneo, cosa que no suele casar con la realidad de una habitación. Al intentar medir los objetos del cuadro, no podremos utilizar la regla graduada. Nuevamente, nuestro cerebro nos hace entender su contenido puesto que ajusta según la similitudes conocidas en nuestra memoria, por ello, entendemos que se trata de una habitación y los objetos usuales contenidos en ella.

Si nos preguntarmos por las imágenes con cierto grado de similitud que utilizará nuestro cerebro para tratar de aproximar el concepto de función, quizasentendamos la esencia de nuestro proyecto. Ahora bien, si entendemos que el profesor puede tener múltiples imágenes asociables al concepto, nos debemos preguntar por la imagen símil que tendrá el estudiante, pues es esencial que el estudiante posea algunas imágenes iniciales para poder entender el concepto. Se tiene que Pero se tiene que algunos investigadores (Sierpinska ;1992, Sfard 1989) analizarón los matices utilizados en las escuelas y en las 
universidades sobre la función, y observaron que se ofrecen versiones distintas del concepto y que esto origina la transparecia de algunas características notables de las funciones. Además, otros investigadores (Nicholas; 1966, Norman; 1992, Goldenberg 1988) muestran un catálogo de dificultades y errores que prenden en el estudiante debido a la utilización esas diferentes versiones matizadas, destacandose que unas versiones facilitan algunos aspectos del concepto y otras interfieren el desarrollo y comprensión (Selden; 1992, Dubinsky y Harel; 1992, Norman; 1992).

Esta situación nos facilitó presentar nuestro proyecto de innovacióncon didáctica que se ha desarrollado como una experiencia continuada en el tiempo durante varios cursos. Con nuestra experiencia experimental hemos intentado curso a curso que este innovador proyecto no caiga dentro de las críticas que indica una referencia de Angel Fiallo: En ocasiones, las experiencias de innovación educativa son similares a los fuegos de artificio. Causa admiración la combinación de tecnologías emergentes, nuevos métodos y resultados asombrosos.

La admiración dura el tiempo que transcurre la presentación, en leer el artículo. Después, nada, nadie lo aplica y lo que puede ser peor: el autor/a de la experiencia de innovación educativa no continúa con ella.

Algunas teorías constructivistas (Piaget, Ausabel y Vygotsky ...) presentan la participación activa del estudiante en su aprendizaje como uno de los puntos importantes de proceso educativo. Otras teorías pedagógicas y psicológicas asumen como básico esa participación práctica. En general, lo que se pretende es cambiar el rol del profesor, dejando de ser el eje sobre el que gira el proceso de enseñanza-aprendizaje. Se quiere marcar una diferencia con la clase magistral donde el profesor es el sujeto y recurso principal para el estudiante, mientras los estudiantes son sujetos pasivos, básicamente. Se desea y postula un profesor guía con un marcado carácter orientador, en definitiva un tutor en el sentido amplio. Un profesor que tiene otra misión esencial como es la de ser un creador de ambientes educativos y cuyo objetivo sea transformar una estrategia para ser un desarrollador de materiales didácticos. Por otras parte se considera que un profesor con esas capacidades le convertirán en un investigador e innovador en su materia. Sin embargo, ese profesor no debe olvidar las facetas activas que debe presentar de pedagogo y, si es necesario, de psicólogo, entre otras cosas.

Se puede decir que añadir a la “definición” de profesor esas capacidades es esencialmente positivo, pero parece descatalogar de forma inmediata a un conjunto de profesionales educativos. Ahora bien, como ser ese tipo de nuevo profesor es bastante difícil entonces se cree, y fomenta, que la participación del profesor en procesos de formación para en adquirir esas capacidades es la solución. El problema surge en el cómo hacer esta formación, puesto que ahí es donde no todos estamos de acuerdo. La razón principal de los desacuerdos es que esas funciones exigibles al profesor dependen esencialmente de la capacidad de comunicación de éste como persona y como experto en la materia que imparte. Esta capacidad comunicativa suele ser obviada conscientemente en los artículos de la literatura docente, de alguna forma se presupone ingenuamente y se universaliza como capacidad comunicativa tradicional o básica: Los profesores saben comunicar con sus estudiantes por el hecho de ser considerado profesor por estos. Sin embargo, no hay método universal de comunicación más allá del lenguaje común, puesto que la faceta comunicativa suele depender de la materia que debe tratar y no es fácilmente generalizable de una materia a otra o de un nivel educativo a otro, ni de un profesor a otro, aunque se crea que se puede hacer. Es 
ingenuo pensar que es fácil generalizar un proceso particular de Enseñanza Primaria a la Enseñanza Secundaria, o de esta a la Enseñanza Universitaria. Esto puede ser muy complejo o cuasi imposible puesto que los actores parten de unas condiciones experimentales iniciales distintas.

Nuestro trabajo se centra en no intentar generalizar procesos de la Enseñanza Secundaria a la Enseñanza Universitaria, si no en generar un cierto lenguaje que tanto el estudiante de secundaria como el estudiante universitario sepan emplear. Esto nos ha facilitado centramos en el concepto de función de una variable real que es común a los dos niveles.

El concepto de función en la formación matemática en la etapa básica es gran importancia, esto lo refleja la National Council of Teacher of Mathematics (NCTM 1989) al señalar que el concepto de función es un "concepto unificador en matemáticas" y que "las funciones forman el lenguaje del cambio y la causalidad es expresado por el simbolismo de las funciones” (NCTM, 2012, p. 51).

La concepción de función reflejada en los libros de texto genera una dificultad didáctica al estudiante (Mesa y Villa 2007; Mesa 2004) por presentar un carácter generalista. Esta dificultad puede ser evitada, al menos, para una gran parte de funciones, que es lo que llevamos a cabo desde nuestro proyecto innovador. Claro que entendemos que voltear los patrones habituales establecidos en los libros de texto tiene su dificultad. Esto mismo ocurre con los patrones empleados por muchos profesores en sus prácticas docentes aun cuando tratan las funciones más elementales. Además, creemos que debemos pensar de un modo creativo para permitirnos diseñar otros modelos educativos del concepto, que catalogamos como un modelo de innovación.

Un proyecto innovador no puede modificar un concepto, el de función, por muy generalista que este sea. Sin embargo, un proyecto puede modificar el proceso educativo de función al que accede el estudiante. En nuestro caso, el cambio se genera haciendo uso de técnicas comunicativas corporales de modo que el estudiante adquiera de forma participativa los elementos básicos icónicos, en cuanto a la forma y modelo. Para ello, establecemos una sintaxis y una semántica para los gestos y los posicionamientos icónicos. Además, al estar situando el foco del proceso en el aprendizaje del estudiante, dando mucha importancia a su participación activa, resulta relativamente importante el marco didáctico en el que se sumerge el método de enseñanza empleado. En este proyecto se nos permite consensuar muchos puntos importantes, desde el mismo juego de formas que se emplea, hasta el tipo de comunicación empleada; unilateral, bilateral y multilateral, de todos a todos. Este proyecto se inició pensando en no permitimos que la inercia de la costumbre y las culturas docentes (matemáticas) asentadas nos atenazaran con repetir esquemas usuales, supuestamente, que podrían ser catalogados gastados y de los que se presume que son sospechosamente inoperantes.

No nos permitimos que el estudiante huya del concepto de función (generalista) sin reconocer la dificultad de su aprendizaje, aunque le hacemos saber que la dificultad de tratar de definir este concepto con precisión trajo como consecuencia grandes controversias en la historia de la matemática entre matemáticos en diversas épocas. Esta dificultad fue un impedimento serio en el desarrollo del cálculo matemático en ciertos momentos. Sin embargo, entendemos que la concepción usual de los profesores 
actuales es consecuencia del desarrollo histórico del concepto y del posicionamiento filosófico de los fundamentos de la matemática (Mesa, 2004) que los matemáticos impulsaron desde 1900.

Desde nuestro punto de vista, se debe entender que el profesor enseñante del concepto de función, está obligado a clarificar el registro de representación semiótica sobre el que se presenta el concepto (Mesa y Villa, 2007; Vargas et. al., 2016). Es decir, los signos empleados, la sintaxis o gramática que combinan esos signos y la semántica relativa a dicha sintaxis. Pero, también, se debe comprender que el estudiante de secundaria no este familiarizado con ese tipo de registro, muy probablemente. Por ello, la necesidad de construir un conjunto de imágenes al que su cerebro pueda acceder.

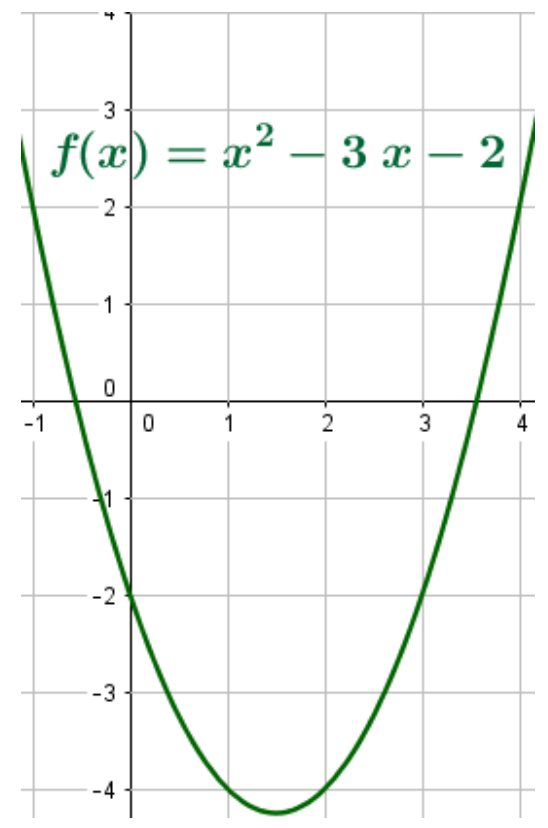

Figura 2: Registros simbólico y gráfico de una función cuadrática.

Del estudiante del Máster de Formación de Profesorado de Enseñanza Secundaria (Matemáticas) se le supone los conocimientos técnicos de la materia, pero sin darse cuenta se encontrará, en su momento, repitiendo el mismo patrón guía de su profesor, y así sucesivamente. El problema se perpetúa mediante repetición generando cultura docente a la vez que el concepto de función se mantiene transparente para el estudiante de secundaria. Al acceder a la universidad, este desconocimiento no se evita de forma eficaz, pues para ellos, una función suele ser una fórmula a la cual hay que manipular según un determinado método algorítmica. Es decir, se hacen “cosas” con funciones pero siguen siendo unas desconocidas en esencia, más cuando aparecen formas, de definir funciones para el estudiante, en la universidad.

\section{Descripción de la experiencia}

Conviene recordar que hasta hace unas pocas décadas, para ser profesor de Matemáticas de Enseñanza Secundaria, simplemente, se requería tener una licenciatura Ciencias Matemáticas, o una licenciatura afín, y una experiencia educativa contrastada o un título de aptitud pedagógica. Actualmente se requiere un Grado del EEES y el título de Máster en Formación del Profesorado de Educación Secundaria. Ahora se postula que la 
formación de un profesor nunca acaba, y se fomenta una actualización constante en formación profesional, para lo cual, se oferta formación continua y formación abierta. Hoy se interpreta que el profesor posea una perspectiva innovadora e investigadora, y esta es la razón por la que ofertamos nuestro modelo innovador de introducir las funciones a los estudiantes del Máster de Formación del Profesorado.

Cabe resaltar que en los últimos años, el profesor universitario debe presentar cierto perfil innovador didáctico y una predisposición de guía del estudiante. Sin embargo, estos perfiles no son reconocidos de forma universal.

\subsection{Los antecedentes}

Este proyecto se remonta a más de 11 años cuando el autor hacía uso de una forma de comunicación gestual en su labor docente. Desde hace siete años (2011) dicha gesticulación se complementó con varios Laboratorios de Simulación Matemática (aplicaciones Geogebra) para generar gráficas y familiarizar al estudiante con las gráficas de algunas funciones. Esto fue destilando de forma simple los iconos básicos que el estudiante adquiere con el desarrollo del proyecto. El periodo de experimentación consta de un mínimo de 10 sesiones de una hora.

La primera experimentación gestual se realizó con los estudiantes no universitarios de UNED, concretamente con el Curso de Acceso para mayores de 25 años del Centro Asociado de Guadalajara. Desde entonces la experiencia ha evolucionado y se ha actualizado, hasta la fecha, para adaptarlo cada vez mejor ese tipo de estudiantes. Un estudiante (no universitario) de este tipo debe adquirir el nivel de Matemáticas de un estudiante de la Enseñanza Secundaria a lo largo de un único curso académico.

La experiencia se extendió en el curso siguiente a estudiantes del Grado de Física, adecuando el conjunto de gestos e iconos debido a que el conjunto de funciones para estos estudiantes es más amplio y reduciendo el número de horas a 5 . La oferta se mantiene en pie puesto que la comunicación corporal mantiene una fuerza semántica que facilita la interpretación de otros conceptos del Análisis, por ejemplo, el límite en el infinito "sin necesidad de cálculos tediosos y repetitivos".

Se aplicó este proyecto con estudiantes del Master de Enseñanza Secundaria de España en diversos cursos (4 horas) y de Ecuador en un curso extraordinario presencial con 6 horas (Matemáticas I, Matemáticas II e Innovación Docente e Investigación Educativa; especialidad de Matemáticas; España, y Ecuador; UNED).

Desde hace cuatro cursos académicos se desarrollan sesiones de hora y media con estudiantes de Enseñanza Secundaria dentro del marco de divulgación y colaboración que realizamos con ColArte bajo el título MatFácil. El tipo de función varía de unos grupos a otros pues están en distinto cursos de Secundaria y Bachillerato. 


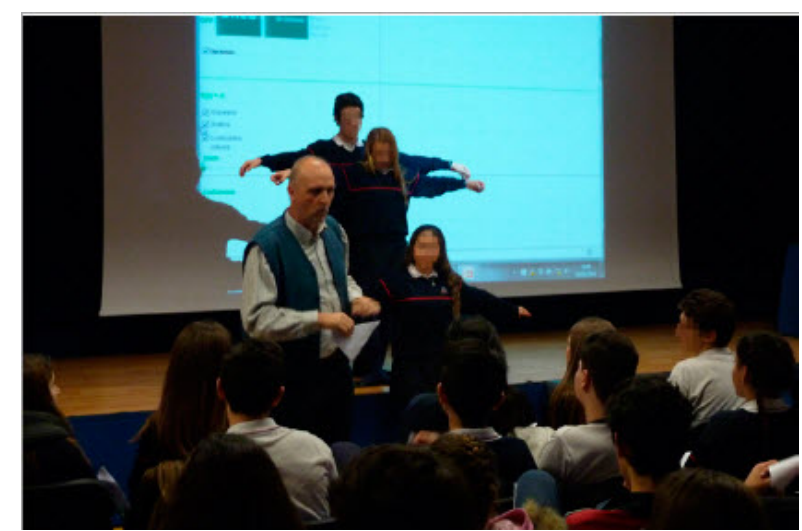

Figura 3: Presentación de MatFácil a estudiante de EESS.

\subsection{Experimento}

La experimentación continuada se realiza amparada en el marco de la teoría de los Espacios de Trabajo Matemático que son una generalización del Espacio de Trabajo en Geometría (Kuzniak, 2006). En esencia, consiste en crear un Espacio de Trabajo Matemático. Esto es, diseñar y desarrollar un entorno de trabajo, organizado y controlado por el profesor para permitir adquirir a sus estudiantes las competencias necesarias para trabajar con las representaciones del concepto función. En los casos de formación de futuros profesores de Matemáticas, se añade una referencia que capaciten a esos profesores como medio para innovar e investigar en Educación Matemática.

- Componentes del plano gesticular:

Todas las funciones son presentadas empleando brazos y cuerpo como elementos de representación. Si bien el proceso se inicia con las funciones polinómicas.

o Las funciones polinómicas de grado 0 se representan extendiendo en horizontal los brazos. La figura básica que sirve de referencia es la función constante $f(x)=1$.

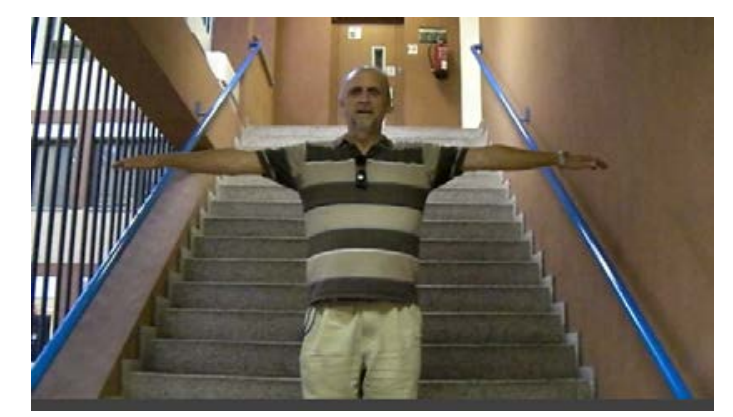

Figura 4: Gestos para funciones constantes.

o Las funciones polinómicas de grado 1 se presentan extendiendo de forma inclinada los brazos. La figura de referencia principal es la función identidad $f(x)=x$.

o Las funciones polinómicas de grado 2 se muestran dando la curvatura adecuada a los dos brazos. En este caso la función primera de referencia es la que corresponde a la parábola $f(x)=x^{2}$. 


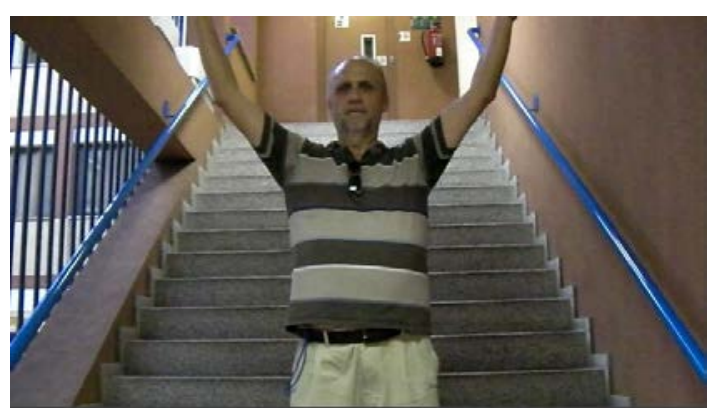

Figura 5: Gestos para funciones constantes y funciones cuadráticas.

0

o Las funciones polinómicas de grado 3 se presentan de forma similar la de las de grado 2 pero cambiando una de las ramas o brazo de posición; de arriba hacia abajo.

Estos primeros elementos sólo requieren la actuación de una única persona puesto que son funciones continuas y definidas para cualquier número real.

o Las funciones raíz cuadrada y raíz cúbica son obtenida por abatimiento de una parábola o parábola cúbica sobre la diagonal (imaginaria).

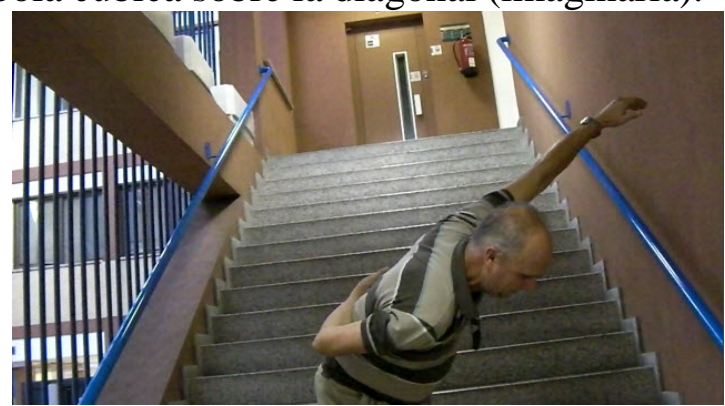

Figura 6: Gestos para representar la función raíz cuadrada.

o Del resto de funciones polinómicas se hace un adecuado listado con los mismos medios pero cambiando lo que hay que ver.

Algunas funciones requiere la actuación conjunta de dos o más personas, por ejemplo, la función $f(x)=\frac{1}{x}$ o la función $f(x)=\frac{1}{x^{2}}$. Para ello es necesario que esas personas se coordinen para representar gestualmente tales funciones. En el caso de las funciones citadas como ejemplo intervienen dos personas, y cada una describe una de las ramas de la función.

- Componentes del plano icónico:

o La representación gráfica generada con alguno de los laboratorios de simulación del experimento. Los iconos se generan al ajustar los gestos a las representaciones gráficas visibles mediante un cañón de proyección o una pizarra digital. 


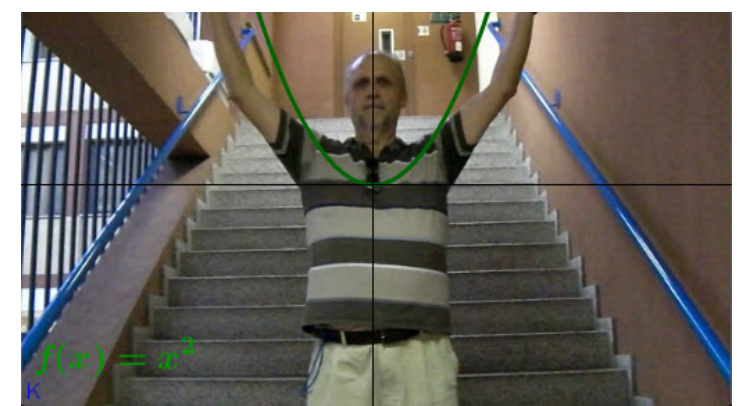

Figura 7: Icono de una función cuadrática.

o Los iconos referentes a familia de funciones. Cada función de una familia tiene la forma del icono canónico y se obtiene por traslación de los puntos básicos del icono o mediante las deformaciones adecuadas.

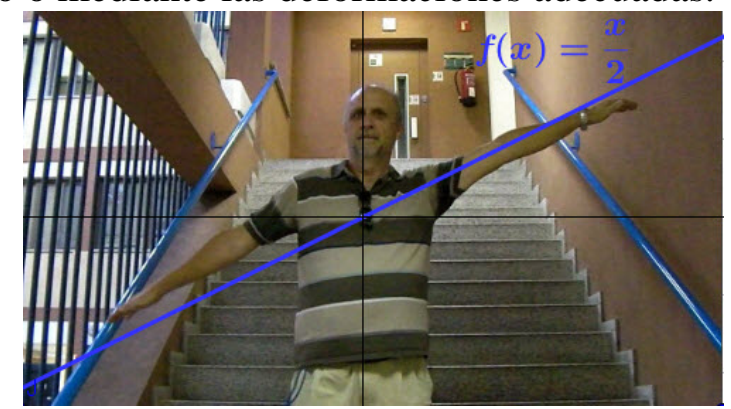

Figura 8: Iconos de la familia de funciones lineales.

o La secuencia de iconos distintos que tiene características similares y la condición diferenciadora de los puntos básicos de un icono.

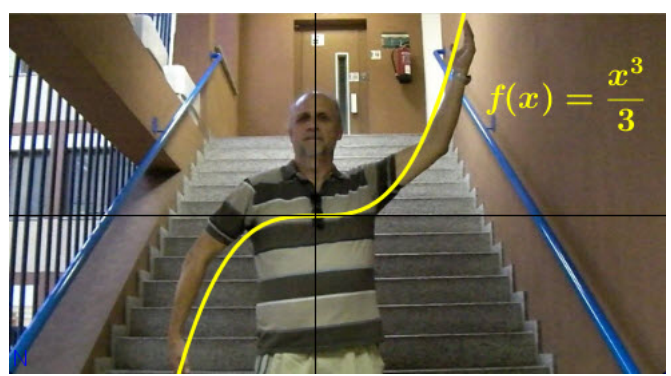

Figura 9: Icono de una función cúbica.

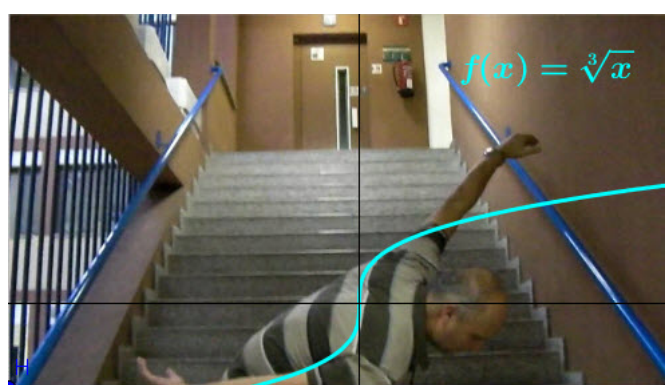

Figura 10: Iconos de una función raíz cúbica obtenida por abatimiento.

\section{Primeros paradigmas en la comunicación gestual de funciones}

Antes de hacer referencia a distintos ejemplos, no conviene recordar hablar de la comprensión de las Matemáticas en el marco de la Educación Matemática no tiene 
sentido: Sin su sistema de signos. (Filloy y Sutherland 1996) y sin sus representaciones. (Duval 1999). Por ello, en este proyecto tenemos que el conjunto de funciones básicas constituyen los signos del lenguaje corporal y tienen su significado en tanto que pertenecen a distintas clases de representaciones gráficas.

El estudiante o el profesor deben traducir directamente la representación simbólica indicada con el gesto que transmite al resto sin necesidad de que exprese palabra alguna. Sin duda, la convergencia entre gestos y gráficas generadas por el laboratorio de simulación sirve para depurar, en cierta medida, la realización de gestos, y con ello, mejorar la transmisión gestual. Sin embargo, una posición corporal relativa a una función no puede ser tan precisa como la respuesta gráfica de un ordenador.

En las figuras de este trabajo se muestra al profesor en medio de unas escaleras para facilitar entender el conjunto de gestos que se realizan. Con algunas funciones se requiere subir o bajar escalones manteniendo el gesto inicial de brazos.

Al estudiante se le dice la expresión de la función de palabra, aunque en algunos casos también se escribe. La idea principal es que no vea la expresión escrita como un conjunto de operaciones que pudiera interpretar como operaciones numéricas. A continuación describimos la secuencia de gestos que facilita la representación corporal de algunas funciones.

Para mostrar cualquier función constante, $f(x)=a$, basta extender los brazos en horizontal, que corresponde a la función $f(x)=0$, y subir o bajar el número de escalones que indique la constante. La tres primeras funciones las gesticula el profesor, y rápidamente, los estudiantes los que generan la conversación con funciones constantes entre risas. Es un primer momento en el cual lo importante no es la exactitud del mensaje, lo importante es la forma gráfica o la pertenencia a una familia de funciones.

Una vez asumido la forma gesticular se pide al estudiante que describa con palabras la gráfica de alguna de estas funciones. La respuesta inmediata es decir que se trata de una recta horizontal, sin nada más. De inicio no indican punto alguno de la recta, pero poco después hacen una descripción precisa: recta horizontal que pasa por el punto $(0, a)$.

Ya hemos dicho que los mensajes se realizan con unas cuantas risas, por lo que, los estudiantes deben superar un primer momento de vergüenza (muy corto), pero rápidamente dejan de ver el distintivo de la constante numérica para quedarse con la forma de la gráfica y el gesto. En este momento se tiene que la posibilidad de realizar los mismos gestos que el profesor es muy motivador y esto empieza a facilitar entender que no imprescindible la precisión de los gestos para comprenderlos. Que algo no sea perfecto no impide entender lo que se dice, simplemente requiere una pequeña adaptación como se indico con el texto imperfecto o con el cuadro de la figura 1.

La adaptación a la interpretación gestual requiere un proceso paso a paso. El texto imperfecto se inicia con una palabra por línea de escritura, luego con otra, más tarde varias palabras, hasta llegar a párrafos enteros. Rápidamente uno está leyendo sin dificultad. Esto mismo es lo que hacemos con las funciones; empezar por las funciones sencillas, consensuar gestos y descripciones. Los gestos para las funciones constantes son asumidos por todos los estudiantes. 
En el caso de las funciones lineales, $f(x)=a x$, se inicia con la posición básica (inicial) de los brazos que representan a una imaginaria diagonal correspondiente a $f(x)=x$. Se gesticula con varios valores (números naturales) para el coeficiente $a$ mostrando el giro de los dos brazos hacía la vertical según el valor considerado desde la posición básica inicial.

Una vez afianzados los gestos con los valores naturales, se presentan las posiciones de los brazos valores de la forma $\frac{1}{n}$ donde $n$ es un número natural. En estos casos se muestra el giro de los dos brazos hacía la horizontal según el valor de $n$ desde la posición básica inicial.

Se continua con los coeficientes negativos haciendo ver la relación entre el gesto de la función a $f(x)=x$ y la de la función a $f(x)=-x$. En general el estudiante entiende los demás casos con coeficiente negativo de una forma natural, llegando a la conclusión que las partes de la gráfica por encima del eje OX pasa a estar por abajo y viceversa. Esta conclusión de simetría respecto al eje OX queda rápidamente asumida en otras funciones polinómicas, que son presentadas posteriormente, sin necesidad de insistir en la cuestión de coeficientes principales negativos.

Nuevamente, una vez asumidos los nuevos gestos se pide al estudiante que describa con palabras la gráfica de alguna de estas funciones. La respuesta inmediata es decir que se trata de una recta inclinada que pasa por el origen. Inicialmente, no indican otro punto de la recta, pero poco después hacen una descripción precisa: recta inclinada que pasa por los puntos $(0,0)$ y $(1, a)$ o algo similar.

La experimentación continua con las funciones afines, $f(x)=a x+b$, siguiendo un proceso similar para cualquiera de ellas. Se inicia presentando el gesto correspondiente a la función $f(x)=x$ si $a>0$ o a la función $f(x)=-x$ si $a<0$. Se continúa haciendo girar los brazos hasta conseguir el gesto correspondiente a la función $f(x)=$ $a x$. Se finaliza subiendo o bajando los escalones correspondientes al coeficiente $b$ manteniendo el gesto que se tenía ya (supuesto que $b$ es un número entero).

Al solicitar la descripción con palabras de la gráfica de alguna de estas funciones, se responde que es una recta paralela a la inclinada correspondiente a función $f(x)=a x$. En este caso se solicita que de determinen dos punto de la recta, y se responde con toda normalidad y sin dificultad: recta inclinada que pasa por el punto $(0, b)$ y el punto $(1, a+b)$ o respuesta similar.

Después de asentados determinada modificación de gestos previos se tratan las funciones cuadráticas (parábolas) partiendo del gesto inicial correspondiente a, $f(x)=$ $x^{2}$, y las funciones cúbicas mostrando los dos tipos diferente que hay, cuyos modelos básicos son , $f(x)=x^{3}, \mathrm{y}, f(x)=x^{3}-x$. Así sucesivamente con el resto de funciones.

\section{Metodología}

Con los primeros ejemplos de funciones se consigue una total integración de los estudiantes al espacio de trabajo matemático. Atienden y quedan a la expectativa del incremento de gestos que describen nuevas funciones. Esto es debido a que la metodología empleada es totalmente práctica y participativa, donde se intenta interpretar cualquier texto y se genera una comunicación bilateral de gestos con las oportunas rectificaciones. Se genera dentro del aula un espacio en el cual la 
comunicación es divertida, sin tener que hacer cálculos ni valoraciones numéricas. Espacio donde el estudiante adquiere un sentido común práctico sobre funciones y donde se siente participe de la imitación de los gestos del profesor cuando éste presenta cada una de las familias de funciones. Además, desde el primer momento adquiere el concepto global y dinámico del concepto de gráfica de una función. Al ser una postura, deja de pensar en operaciones numéricas y tabulaciones de puntos de la gráfica, pues esos son elementos añadidos que no son estrictamente necesarios para entender de qué función se trata en cada momento.

En los momentos de dificultad, o de duda, a la hora de elegir un gesto para una nueva función, o ratificar el texto elegido, se hace uso de las representaciones que hace la aplicación del ordenador. Estas sólo son necesarias para mostrar de una forma rápida la variedad de formas en que se puede presentar cada familia de funciones del mismo tipo.

Tanto el profesor como el estudiante trabaja los gestos de forma directa, es decir, de parte del registro de representación simbólico hasta generar una secuencia de gestos que son refutados posteriormente con el laboratorio de simulación. También, trabaja la forma inversa, es decir, dado una situación gestual, se postulan posibles representaciones simbólicas. En este proceso se comprueba la forma de la representación gráfica y se postula que los gestos y las expresiones explícitas no están relacionados de forma unívoca, que son necesarios algunos datos más de la función gesticulada que se incorporan de palabra.

Con esta clase de representación se genera un “dialogo de locos” que permite pactar aciertos y engaños, y crear un juego que fácilmente se apodera de la adquisición de formas para entrar en el juego.

\section{Resultados}

En algunas ocasiones la excesiva manipulación algebraica que se hace en la enseñanza del concepto de función por parte del profesor hace que sus estudiantes entonen el estribillo de una canción de Celtas Cortos:

$$
\begin{aligned}
& \text { Cuéntame un cuento } \\
& y \text { veras que contento } \\
& \text { me voy a la cama } \\
& \text { y tengo lindos sueños... }
\end{aligned}
$$

En nuestro proyecto resulta que tras la fase de juego y del reconocimiento de formas, no sólo se dispone de una concepción dinámica del concepto de función, si no que se tiene la posibilidad de hacer frente a conceptos matemáticos abstractos y profundos de una forma muy visual. Por ejemplo:

$1^{\circ} \mathrm{La}$ “abertura” de una parábola sólo depende del término cuadrático (abierta arriba o abajo, más estrecha o ancha que la icónica), lo único que cambia es dónde se sitúa el vértice de dicha parábola.

$2^{\circ}$ Las operaciones de funciones, dejan de ser simples operaciones de polinomios para poder entender la forma en la que se combinan los gestos y las formas. Por ejemplo sumar dos gestos rectilíneos genera un gesto rectilíneo, o sumar un gesto horizontal y uno rectilíneo simplemente genera una traslación en el eje vertical. 
$3^{\circ}$ Se puede introducir al análisis del límite en el infinito (positivo o negativo) tan sólo haciendo ver que esa cuestión se transforma en saber cómo se comporta la gráfica de la función en el infinito. Para ello, se dispone del modo gesto que explicita la situación en el más allá.

$4^{\circ}$ Las funciones definidas a trozos son interpretadas de una forma fácil, siempre y cuando sean continuas. En caso contrario se requiere la participación de más estudiantes. Esto permite introducir la concepción de continuidad en un punto de los límites laterales y límites.

Y casi todo lo dicho sin tener que hacer “cálculos" muy pesados con un potente aparataje algebraico. Las formas están antes que los cálculos y los cálculos aparecen cuando no se puede tener seguridad sobre las gráficas.

\section{Conclusiones}

Esta forma de presentar las funciones es muy sencilla y muy operativa, pues permite al estudiante afianzarse en las formas de las funciones más sencillas que se le presentan en su estudio de la materia. Aunque no es fácil que el estudiante se implique en el juego al principio, pues se generan risas y comentarios, la realidad se impone cuando todos se proponen el reto de comunicar con gestos la función que tratan. En los casos de estudiantes de EESS, la acción grupal favorece la integración de los saberes individuales, se reconoce como un juego de todos con todos y dentro de las matemáticas.

\section{Referencias}

Kleiner, I. (1989). Evolution of the function concept: A brief survey [Evolución del concepto de función: una breve encuesta]. The College Mathematics Journal, 20(4), 282-300.

Kuzniak, A. (2006), Paradigmes et espaces de travail géométriques. Éléments d’un cadre théorique pour l'enseignement et la formation des enseignants en géométrie, Canadian Journal of Science, Mathematics and Technology Education, 6(2), 167-188.

Luzín, N. (1998). En: Ferreiros, J. (2003). Historia del concepto de función. La Gacete de la RSME, 6(21): 413-436.

Mesa, V. (2004). Characterizing Practices Associated with Functions in Middle School Textbooks: An Empirical Approach [Caracterización de las prácticas asociadas con las funciones de los libros de texto de las escuelas intermedias: un enfoque empírico]. Educational Studies in Mathematic, 56, 255-286.

Mesa, Y.M. y Villa, O.J. (2007). Elementos históricos, epistemológicos y didácticos para la construcción del concepto de función cuadrática. Fundación Universitaria Católica del Norte. 
National Council of Teachers of Mathematics. (2012). Professional Standards' for Teaching Mathematics [Normas profesionales para la enseñanza de las matemáticas]. Reston, VA: The Author.

National Research Council. (1989). Everybody counts: A report to the Nation on the Future of Mathematics Education [Todo el mundo cuenta: Un informe a la nación sobre el futuro de la educación matemática]. Washington, D. C: National Academy Press. 